\title{
RESÍDUOS DE PORCELANATO: UMA REVISÃO NA LITERATURA INTERNACIONAL
}

\author{
Samuel Jônatas de Castro Lopes, Centro Universitário Santo Agostinho ${ }^{1}$ \\ Mikael de Sousa Marques, Centro Universitário Santo Agostinho² \\ Emmanuel Jesus Olimpio Vieira, Centro Universitário Santo Agostinho ${ }^{3}$
}

\section{RESUMO}

A construção civil é um dos principais responsáveis pela geração de resíduos sólidos. Dessa forma, faz-se necessário buscar soluções viáveis que busquem reduzir o impacto ao meio ambiente causado pelo descarte incorreto de tais resíduos além da possibilidade de se obter possíveis ganhos econômicos. Com o processo de modernização da construção civil, novas tendências e materiais surgiram e o porcelanato se solidificou no mercado. Este artigo objetiva analisar a literatura internacional sobre resíduos de porcelanato em função da geração e reutilização de resíduos sólidos. Uma revisão da literatura foi executada na base do Web of Science, com objetivo de demonstrar como este tema está sendo debatido no cenário internacional. Pode-se observar que esse tema se trata de um assunto recente, onde o artigo mais antigo encontrado foi no ano 2002 sendo que as principais abordagens teóricas foram reutilização do resíduo de porcelanato no seu processo de fabricação e ainda como substituto de outros materiais da construção civil.

Palavras-Chave: Construção Civil. Porcelanato. Reutilização.

\section{INTRODUÇÃO}

O crescimento industrial a partir da Revolução Industrial e com o avanço tecnológico vem gerando um aglomerado de resíduos, podendo provocar poluição ambiental e potencial risco à saúde (BRASILEIRO; MATOS, 2015). O setor da construção civil ao mesmo tempo em que cresce, consome mais recursos naturais e muitas vezes por conta da falta de gerenciamento produz uma grande quantidade de resíduos sólidos (CALDEIRA; SERRADILHA; RODRIGUES, 2016). Conforme a Resolução no 307 do CONAMA os resíduos da construção civil são derivados das construções, reformas, reparos e demolições de obras de construção civil (BRASIL, 2018).

\footnotetext{
${ }^{1}$ Graduado em Engenharia Civil, Centro Universitário Santo Agostinho, samueljonatas99@hotmail.com.

${ }^{2}$ Graduado em Engenharia Civil, Centro Universitário Santo Agostinho,mikael_sm@hotmail.com.

${ }^{3}$ Graduando em Engenharia Civil, Centro Universitário Santo Agostinho, ejje.vieira@gmail.com. ISBN: 978-65-80968-11-4
} DOI: $10.17648 /$ cbcs-2019-110663 


\section{CONWEESSOO CIENCIAESOCIEDADE

ANAIS CBCS 2019 | 3 a 5 de outubro de 2019 | Centro Universitário Santo Agostinho - Teresina - P|

De acordo com Kuzma et al. (2017) a forma de descarte dos resíduos sólidos influencia diretamente a maneira de conduzir uma organização, vez que não impactam somente a sociedade e o meio ambiente, mas também o ambiente econômico empresarial.

A busca pelo equilíbrio entre desenvolvimento econômico e a proteção ambiental exige que as forças produtivas e as formas de produção estejam alinhadas numa mesma direção, a consciência ambiental (SLOMSKI et al., 2017).

Desta forma, o envolvimento das organizações em questões socioambientais pode significar oportunidade de negócios, por meio de uma reorganização empresarial em torno dos processos, tecnologias limpas e o reaproveitamento de resíduos, a fim de obter uma melhor imagem institucional, ganhos em produtividade e uma melhor relação com a comunidade, grupos ambientais e órgãos governamentais (CLARO; CLARO 2014).

Diante desse contexto, esse trabalho tem como objetivo verificar a produção científica internacional quanto à geração e reutilização dos resíduos de porcelanato. Para isso, foi desenvolvido uma revisão na literatura internacional, tendo como base de consulta artigos publicados em periódicos indexados ao Web of Science.

\section{MATERIAL E MÉTODOS}

O presente trabalho é caracterizado como uma revisão da literatura internacional, onde deverá apresentar um protocolo claro para procurar sistematicamente bases de dados definidas ao longo do período de tempo definido, com ponderações bem esclarecidas para a inclusão ou exclusão de pesquisas, bem como avaliar os resultados obtidos.

Para a pesquisa de artigos foi utilizada os locais de busca da base de dados do Web of Science. A busca foi iniciada a partir das palavras-chave na literatura que estão relacionadas à base "Resíduo + Porcelanato". A partir delas foi construído o seguinte termo de busca: (Resíduo de Porcelanato). O tempo não foi restringindo, com objetivo de encontrar o maior número de trabalhos sobre esse tema, logo foi utilizado todo o alcance das bases de dados (1945-2018). 


\section{CONGEESSO CIENCIAESOCIEDADE \\ Inovação, Diversiliaile e Sustentalililitaile}

ANAIS CBCS 2019 | 3 a 5 de outubro de 2019 | Centro Universitário Santo Agostinho - Teresina - PI

A fim de se obter uma pesquisa mais eficiente, utilizaram-se os seguintes tipos de filtro:

- $\quad$ Base de dados: Web of Science Core Collection

- Categorias da pesquisa: "Materials Science Ceramics", "Construction Building Technology" and "Green Sustainable Science Technology".

- $\quad$ Tipos de documentos: Article

Inicialmente o refinamento da pesquisa foi feita através da leitura dos resumos, sendo considerados como pontos de exclusão:

- $\quad$ Exclusão dos trabalhos que tratavam de optimization of the technological properties of porcelain, waste ceramic in asphalt, microstructure of porcelain, Synthesis of fe-mn, pigments ceramic, additive on microstructure, re-use of mud, use of wastes in porcelain tile, analysis of leaching and solubility, waste of colemanite por não se configurarem como objeto de estudo.

Após a aplicação desses filtros chegou-se a cinco artigos que se adequaram ao objetivo desse estudo. Esses artigos que foram filtrados tiveram uma leitura completa, para se observar como os pesquisadores internacionais discorrem sobre esse tema de resíduos de porcelanato e seu reaproveitamento no âmbito da construção civil.

\section{RESULTADOS E DISCUSSÃO}

A análise dos dados foi classificada em duas etapas. A primeira etapa, chamada de análise descritiva tiveram para a classificação das pesquisas as categorias: ano de publicação e principais títulos da fonte. A segunda etapa da análise, classificada como exploratória compreende na identificação das principais temáticas que compõem os artigos que foram analisados, assim como as abordagens teóricas utilizadas para discorrer sobre o tema.

As pesquisas foram executadas em maio de 2018 e a princípio foram encontradas 28 pesquisas. Após a aplicação dos filtros sobre categorias da pesquisa e tipos de documentos chegouse a cinco pesquisas. Após a leitura dos resumos constatou-se que seriam aproveitadas cinco 


\section{conghESSO CIENCIAESOCIEDADE

ANAIS CBCS 2019 | 3 a 5 de outubro de 2019 | Centro Universitário Santo Agostinho - Teresina - PI

pesquisas que reutilizam os resíduos de porcelanato. Desses cinco artigos, a categoria que se destacou foi a de Materials Science Ceramics, conforme sumarizado na Imagem 1.

IMAGEM 1. Classificação dos artigos por categoria

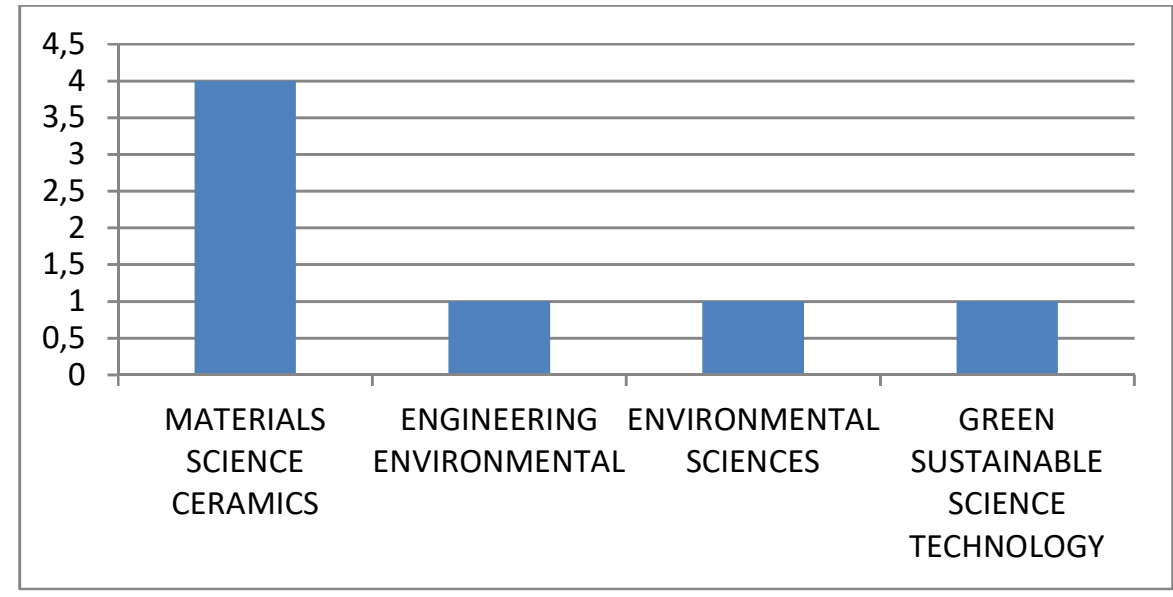

Fonte: Web of Science, 2018.

A análise descritiva dos artigos consiste em classificar nas categorias de nacionalidade dos autores, ano de publicação dos autores e principais títulos da fonte. Podemos concluir com os dados da Imagem 2, que o tema de reaproveitamento vem ganhando força no último ano, em 2017 teve $60 \%$ das publicações com quatro artigos, ou seja, esse é um tema que está ganhando força no cenário mundial. Mesmo não tendo sido colocado restrições quanto à questão do tempo o primeiro artigo publicado da amostra foi no ano de 2002.

IMAGEM 2. Ano dos artigos analisados

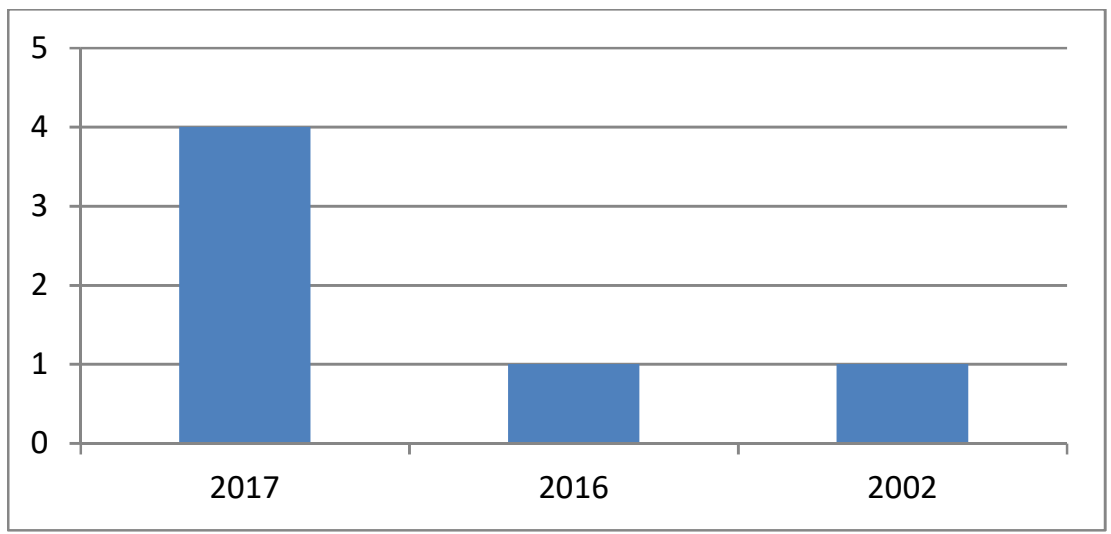

Fonte: Web of Science, 2018. 


\section{CONQGEESSO CIENCIAESOCIEDADE

ANAIS CBCS 2019 | 3 a 5 de outubro de 2019 | Centro Universitário Santo Agostinho - Teresina - PI

Dentre os autores no cenário mundial que estão pesquisando sobre o tema de resíduo do porcelanato, destacam-se Lin $\mathrm{Ht}$ e Xiong H. Ao analisar também por meio da categoria nacionalidade dos autores, a China ficou à frente dos demais países com duas pesquisas.

Em relação aos principais Journals que publicam sobre o tema podemos verificar que o Journal of the European Ceramic Society está à frente dos demais. Esses títulos da fonte podem ser mais bem analisados na Imagem 3.

IMAGEM 3. Principais Journals contidos na amostra

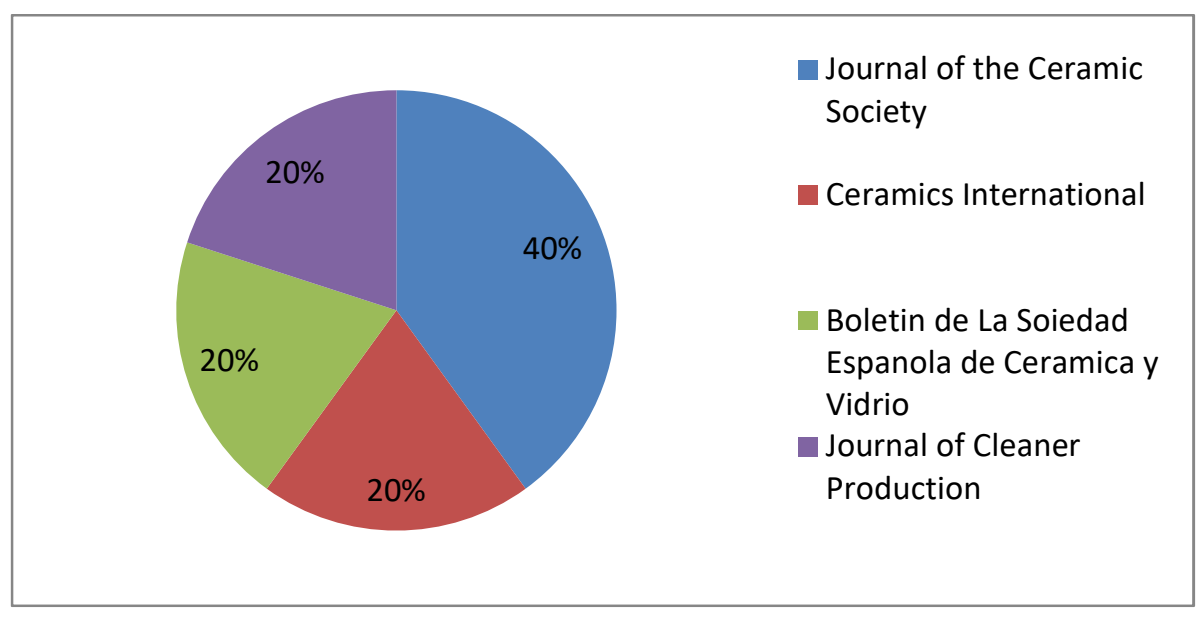

Fonte: Web of Science, 2018.

Na etapa de análise exploratória dos artigos, Xi et al. (2017), afirma em seu estudo que o pó do resíduo de porcelanato pode ser utilizados como matéria prima, e que com adição de carboneto de silício e silício em pó promovem a redução de espuma no processo de reciclagem direta do porcelanato, otimizando o processo e viabilizando sua reciclagem no mundo. Ainda segundo o autor a necessidade de procurar soluções para reciclar os resíduos de porcelanato já é uma realidade.

A substituição de resíduo de porcelanato pelo cimento ou areia na fabricação de blocos de pavimentação é tecnicamente viável. Essa afirmação se deve aos testes que foram realizados onde se possibilita substituir $30 \%$ do agregado fino ou $20 \%$ do cimento, entretanto quando foi substituído o resíduo de porcelanato pela areia obtiveram-se melhores resultados de resistência, entretanto, 
ANAIS CBCS 2019 | 3 a 5 de outubro de 2019 | Centro Universitário Santo Agostinho - Teresina - P|

nos dois casos foram alcançadas as exigências de padrão que é de $50 \mathrm{MPa}$ para o trafego de veículos pesados (PENTEADO; CARVALHO; LINTZ, 2016).

De acordo com Mestre et al. (2002), no processo de fabricação do porcelanato se origina lamas residuais que promovem impactos ambientais no seu descarte ao meio ambiente, com base nessa realidade foi desenvolvido um trabalho de reaproveitamento dessa lama no processo de fabricação mesmo que leve a uma mudança de cor do porcelanato.

\section{CONCLUSÕES}

Diante das pesquisas e da revisão literária internacional, foi possível observar que a utilização de resíduos de porcelanato pode contribuir ambientalmente, assim como melhorar os ganhos empresariais com a reutilização desses resíduos e redução dos custos de matéria prima.

Foi possível observar que o tema teve um crescimento nas pesquisas durante o ano de 2017 por conta da responsabilidade sobre a questão ambiental que está se intensificando a cada dia, além de que a quantidade de porcelanato produzida só aumenta no mundo e juntamente com esse crescimento a geração de resíduos está numa função crescente, então surge à necessidade de encontrar soluções para gerir esses resíduos buscando uma maneira de reaproveitá-los.

Os pesquisadores que se destacaram sobre esse tema em primeiro lugar foram os chineses, vindo em segundo lugar os brasileiros, turcos e espanhóis. Esse protagonismo da China se destaca em várias áreas de pesquisa e esse tema de resíduos de porcelanato tem muito importante para esse país pelo fato da China propor políticas de preservação ao meio ambiente.

Como restrição dessa pesquisa as buscas foram limitadas a base de dados do Web of Science, o que pode ter reduzido a quantidade real de pesquisas feitas no cenário internacional e com isso diminuído a confiabilidade dos resultados. Por outro lado, essa pesquisa englobou artigos publicados nos principais Journals do cenário internacional da área, mantendo um alto poder qualitativo para tal estudo. 


\section{GONGSESSOCOENCIA:SOCIEDADE}

ANAIS CBCS 2019 | 3 a 5 de outubro de 2019 | Centro Universitário Santo Agostinho - Teresina - P|

\section{REFERÊNCIAS}

BRASIL. Ministério do Meio Ambiente. Conselho Nacional do Meio Ambiente, CONAMA. Resolução CONAMA no 307, de 5 de julho de 2002. Estabelece diretrizes, critérios e procedimentos para a gestão dos resíduos da construção civil. Brasília, DF, 2002. Disponível em:

http://www2.mma.gov.br/sitio/ Acesso em: 21 mai. 2018.

BRASILEIRO, L. L.; MATOS, J. M. E. Revisão bibliográfica: reutilização de resíduos da construção e demolição na indústria da construção civil (Literature review: reuse of construction and demolition waste in the construction industry). Cerâmica, v. 61, p. 178-189, 2015.

CALDEIRA, A. C. V.; SERRADILHA, V. H.; RODRIGUES, P. S. H. Utilização de resíduo de bloco estrutural de concreto na substituição parcial e total de agregados e aglomerantes em traço de concreto estrutural. Revista Engenharia em Ação UniToledo, v. 1, n. 01, 2016.

CLARO, P. B. O.; CLARO, D. P. Sustentabilidade Estratégica: existe retorno no longo prazo? Revista de Administração de Empresas, 49(2), 291-306, 2014.

KUZMA, E. L.; LUZ, T. E.; NOVAK, M. A. L.; NAVARRO, R. M. Tratamento de resíduos sólidos e efluentes: uma análise de custos em empresas de revenda de combustível. Revista Metropolitana de Sustentabilidade, v. 7, n. 3, 2015.

MESTRE, S; SANCHEZ, E.; GARCIA, J.; SANCHES, J.; SOLER, C.; PORTOLES, J.; SALES, J. Utilización de la teoría de Kubelka-Munk para optimizar el reciclado de residuos crudos de grés porcelánico .

Boletin de la Sociedad Española de Cerámica y Vidrio, v. 41, n. 5, p. 429-435, 2002.

PENTEADO, C. S. G.; DE CARVALHO, E. V.; LINTZ, R. C. C. Reusing ceramic tile polishing waste in paving block manufacturing. Journal of Cleaner Production, v. 112, p. 514-520, 2016.

SLOMSKI, V.; SLOMSKI, V. G.; BORGES, T. N.; PEREIRA, A. C.; TASIMA, E. C. A adequação da evidenciação ambiental quanto à Política Nacional de Resíduos Sólidos (PNRS), no relatório de sustentabilidade de uma empresa do setor agropecuário integrante da carteira ISE. Seminários em Administração. XX SEMEAD, nov.2017.

XI, X.; XIONG, H.; ZOU, C.; ZUO, F.; HUANG, R.; LIN, H.-T. Oxidation protection of SiC in porcelain tile ceramics by adding Si powder. Journal of the European Ceramic Society, v. 37, n. 7, p. 27532756, 2017. 